Г. Г. ХУБУААВА, К. А. КОЗАОВ, А. Н. ШИШКЕВИЧ, С. С. МИХАЙАОВ, Е. Ю. БЕССОНОВ, П. А. ТОКАРЕВ, $\triangle$. А. ПАЧКОВ, Е. Е. БОБРОВСКАЯ

Предикторы репердузионного синдрома миокарда: современный взгяя на вопрос и актуамьные проблемы. Часть 2: феномен невосстановленного коронарного кровотока, или феномен no-reflow (обзор митературы)

Федеральное государственное бюджетное военное образовательное учреждение высшего образования «Военномедицинская акахемия имени С. М. Кирова» Министерства обороны Российской Федерации, Санкт-Петербург, Россия 194044, Санкт-Петербург, ул. Акацемика Аебедева, А. 6

E-mail: ewgenijbessonov@yandex.ru

\title{
Резюме
}

Статья поступила в редакциюю 27.01.21 г.; принята к печати 28.05.21 2.

Несмотря на то, что феномен невосстановленного коронарного кровотока (no-reflow) давно известен, до сих пор нет однозначного мнения на счет предикторов, а соответственно, и групп риска его возникновения. Это препятствует прогнозированию дальнейшего течения заболевания и исследованию эффективности терапевтических и хирургических способов предотвращения последствий реперфузии у пациентов с инфарктом миокарда с подъемом сегмента ST, что, в свою очередь, ухудшает послеоперационный и отдаленный прогноз у данной группы больных. Для поиска исследований по этой проблеме мы использовали поисковые системы E-lilbrary, Google Scholar и Pubmed. В статье представлены данные исследований, освещающих предикторы феномена no-reflow, проведен их анализ. Также описаны сложности их выявления, поднята проблема диагностического подхода и формирования выборки для исследований. Дан обзор исследований, посвященных выявлению предикторов феномена no-reflow с помощью оптической когерентной томографии и внутрисосудистого ультразвукового исследования.

Ключевые слова: реперфузионный синдром миокарда, инфаркт миокарда с подъемом сегмента ST, феномен невосстановленного коронарного кровотока, по-reflow, повреждение миокарда, рентгеноэндоваскулярная хирургия

Для цитирования: Хубулава Г. Г., Козлов К. Л., Шиикевич А. Н., Михайлов С. С., Бессонов Е. Ю., Токарев П. А., Пачков Д. А., Бобровская Е. Е. Предикторы реперфузионного синдрома миокарда: современный взгляд на вопрос и актуальные проблемы. Часть 2: феномен невосстановленного коронарного кровотока, или феномен по-reflow (обзор литературы). Регионарное кровообращение и микрочиркуляиия. 2021;20(3):4-10. Dоі: 10.24884/1682-6655-2021-20-3-4-10.

UDC 616-005.8

DOI: $10.24884 / 1682-6655-2021-20-3-4-10$

G. G. KHUBULAVA, K. L. KOZLOV, A. N. SHISHKEVICH, S. S. MIKHAILOV, E. YU. BESSONOV, P. A. TOKAREV, D. A. PACHKOV, E. E. BOBROVSKAYA

\section{Predictors of myocardial reperfusion syndrome: a modern view of the issue and current problems. Part 2: no-reflow phenomenon (literature review)}

Military Medical Academy, Saint Petersburg, Russia

6, Academiva Lebedeva street, Saint Petersburg, Russia, 194044

E-mail: ewgenijbessonov@yandex.ru

Summary

Received 27.01.21; accepted 28.05.21

Despite the fact that the phenomenon of unrecovered coronary blood flow (no-reflow) has long been known, there is still no unanimous opinion about the predictors and, accordingly, the risk groups for its occurrence. This prevents predicting the further course of the disease and investigating the effectiveness of therapeutic and surgical methods for preventing the consequences of reperfusion in patients with ST-segment elevation myocardial infarction, which in turn worsens the postoperative and long-term prognosis in this group of patients. We used the search engines E-library, Google Scholar and Pubmed to search 
for studies on this issue. The article presents and analyzes research data covering the predictors of the no-reflow phenomenon. The difficulties in identifying them are also described, the issue of a diagnostic approach ans sampling for research is raised. A review of studies dedicated to the identification of predictors of the no-reflow phenomenon using optical coherence tomography and intravascular ultrasound is given.

Keywords: myocardial reperfusion syndrome, ST-elevation myocardial infarction, no-reflow phenomenon, myocardial injury, X-ray endovascular surgery

For citation: Khubulava G. G., Kozlov K. L., Shishkevich A. N., Mikhailov S. S., Bessonov E. Yu., Tokarev P. A., Pachkov D. A., Bobrovskaya E. E. Predictors of myocardial reperfusion syndrome: a modern view of the issue and current problems. Part 2: no-reflow phenomenon (literature review). Regional hemodynamics and microcirculation. 2021;20(3):4-10. Doi: 10.24884/1682-6655-2021-20-3-4-10.

\section{Введение}

Феномен невосстановленного коронарного кровотока (ФНКК), или феномен no-reflow, является постреперфузионным осложнением и характеризуется отсутствием восстановления венечного кровотока на эпикардиальном, субэпикардиальном и (или) микрососудистом уровне, при отсутствии диссекции стенки артерии или тромбоза $[1,2]$. В число возможных механизмов, способствующих развитию ФНКК, входят эмболизация капилляров, вызванная микротромбами и липидными фрагментами атеросклеротической бляшки; отек и контрактура клеток миокарда, сжимающие микрососудистое русло; отек эндотелия, спазм и дисфункция капилляров; накопление нейтрофилов и тромбоцитов, способствующее воспалению и обтурации микроциркуляторного русла; ишемическое и реперфузионное повреждение; индивидуальная предрасположенность вследствие генетических факторов, анатомических особенностей коронарного кровообращения и наличия сопутствующих патологий [3-6].

Целью нашего обзора является анализ современной литературы и синтез данных, касающихся данной проблемы.

\section{Проблема неоднородности}

\section{Аиагностического подхода}

На наш взгляд, прежде чем речь пойдет о предикторах ФНКК, следует осветить проблему диагностического подхода к верификации данного проявления реперфузионного синдрома. Феномен no-reflow верифицируется на основании инвазивных (степень эпикардиального кровотока по шкале Thrombolysis in Myocardial Infarction (TIMI)<3, степень «прокрашивания» миокарда по шкале Myocardial blush grade $(\mathrm{MBG})<2$, в том числе при TIMI=3) и неинвазивных (микроваскулярная обструкция по данным контрастной эхокардиографии миокарда, магнитной резонансной томографии с контрастированием, при отсутствии разрешения сегмента ST) методов обследования пациента непосредственно после реперфузии. Следует отметить, что частота ФНКК у пациентов с ИМпST варьируется от 5 до 30 \% при верификации с использованием показателей эпикардиального кровотока и до 60 \% при использовании эхокардиографии миокарда с контрастом и магнитно-резонансной томографии, что говорит о явном преимуществе данных инструментов в диагностике микроваскулярной обструкции (MBO) в сравнении с ангиографическими методами $[7,8]$. Однако изза дороговизны и сложности использование данных методов доступно далеко не всем исследователям, и они вынуждены полагаться на суррогатные, весьма субъективные маркеры ФНКК, не полностью оценивающие МВО. Несмотря на то, что статус эпикардиального и субэпикардиального кровотока после реперфузии как факторы летального исхода, жизнеугрожающих аритмий, негативного ремоделирования левого желудочка и прогрессирования сердечной недостаточности подчеркивается многими исследователями [9-14], наличие МВО остается не зависимым от статуса кровотока в эпикардиальных и субэпикардиальных артериях предиктором неблагоприятных последствий для пациента $[15,16]$. Одним из простых и доступных маркеров микроваскулярной обструкции является отсутствие снижения амплитуды сегмента ST. Исследование R. Nijveldt et al. [17] продемонстрировало корреляцию МВО с неполным разрешением сегмента ST, независимо от показателей шкал, оценивающих эпикардиальный и субэпикардиальный кровоток после реперфузии, a G. Niccoli et al. [18] доказали, что комбинация ангиографии и электрокардиографии позволяет значительно улучшить оценку оптимальности реперфузии миокарда. Помимо этого, в одном из своих систематических обзоров G. Niccoli et al. подчеркнули прогностическую значимость МВО (рисунок).

На рисунке, $a$ - кривая выживаемости Каплана Мейера, показывающая при длительном наблюдении (100 месяцев), что пациенты в группе с TIMI $\leq 2$ имели значительно более высокую частоту сердечной смерти (\%) по сравнению с пациентами с более низкими степенями TIMI (P<0,0001) [9]. На рисунке, б - кривая выживаемости Каплана - Мейера показывает кумулятивную частоту нежелательных явлений (\%) в соответствии со степенью MBG среди пациентов с разрешением сегмента ST и без него в течение 1 года наблюдения $(\mathrm{P}<0,01)$. В частности, среди пациентов со снижением амплитуды сегмента ST $<70$ \% кумулятивная частота нежелательных явлений составила $10,1 \%$ для степени MBG 0/1 и 6,3 \% для степени MBG 2/3. Среди пациентов со снижением амплитуды сегмента ST>70 \% совокупная частота событий составила 5,1\% для степени MBG 0/1 и 1,2\% для степени 2/3 [19]. На рисунке, в - кривая выживаемости Каплана - Мейера, показывающая комбинированную выживаемость без событий (\%) у пациентов с и без МВО, после реперфузии, верифицируемую с помощью контрастной эхокардиографии миокарда. Пациенты без МВО демонстрируют более высокую совокупную 5-летнюю комбинированную частоту сердечно-сосудистых событий (Р<0,0001), чем пациенты без микрососудистой дисфункции [20]. На рисунке, г- кривая выживаемости Каплана - Мейера 

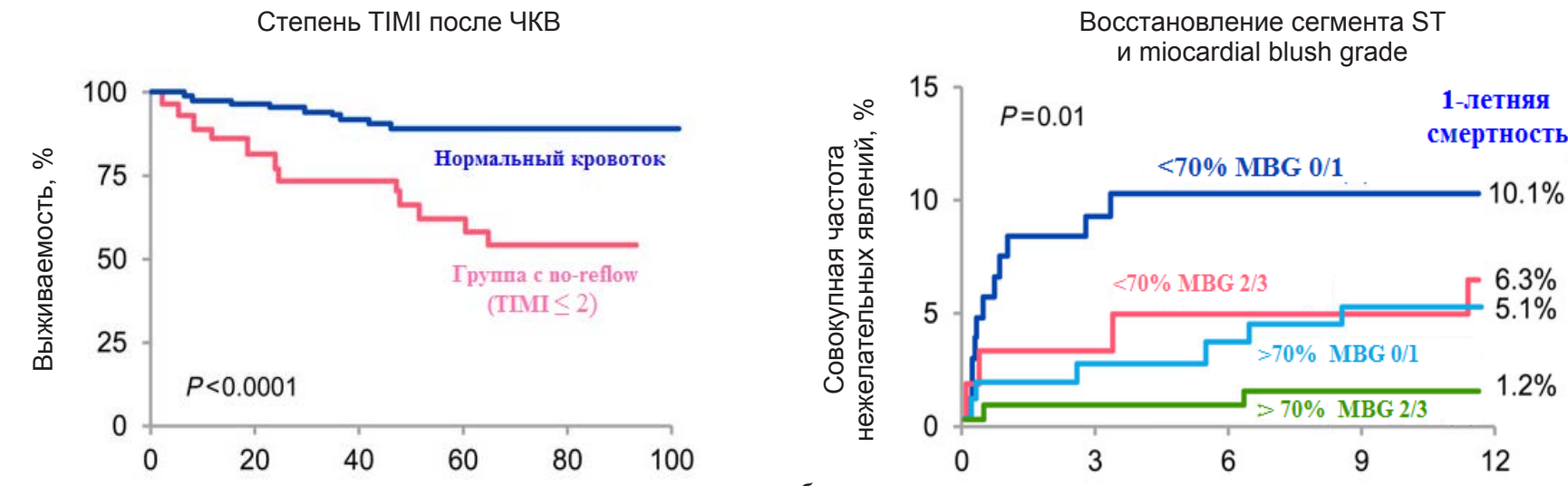

Контрастная ЭхоКГ

6
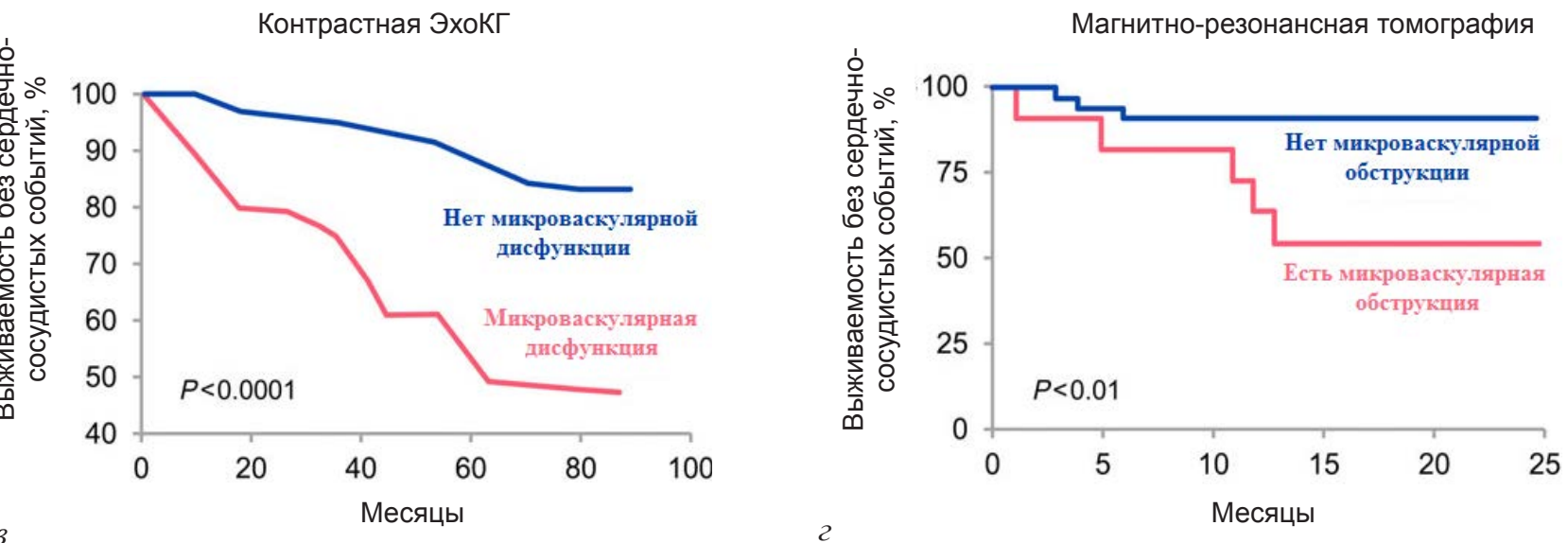

Прогностическая роль коронарной микрососудистой обструкции. Адаптированный перевод: G. Niccoli et al. [7]

The prognostic role of coronary microvascular obstruction. Adapted translation: G. Niccoli et al. [7]

показывает комбинированную выживаемость без сердечно-сосудистых событий (\%) у пациентов с МВО и без нее, после реперфузии, на основании данных магнитно-резонансной томографии. У пациентов с МBO более высокая совокупная 2-летняя комбинированная частота сердечно-сосудистых событий $(\mathrm{P}<0,001)$, чем у пациентов без MBO [21].

Однако в большинстве современных исследований ФНКК верифицируется только на основании показателей эпикардиального и субэпикардиального кровотока, а работы, диагностирующие ФНКК с помощью более совершенных методов и в совокупности эпикардиального кровотока вместе с уменьшением сегмента ST, немногочисленны и включают в себя небольшие выборки пациентов.

\section{Предикторы феномена невосстановленного} коронарного кровотока в мировой митературе

Из-за многофакторного патогенеза и диагностической неоднозначности поиск предикторов ФНКК остается сложной и не решенной проблемой по сей день. Метаанализ, проведенный J. K. Fajah et al. [22], включающий в себя 27 исследований, показал, что риск возникновения ФНКК связан с:

- пожилым возрастом;

- мужским полом;

- сахарным диабетом;

- повышенным уровнем глюкозы крови;

- большим временем боль-баллон;

- классом острой сердечной недостаточности по Killip $\geq 2$;
- высокой частотой сердечных сокращений (ЧСС);

- высоким уровнем креатинина крови;

- уменьшением фракции выброса левого желудочка;

- степенью коллатерального кровотока по Rentrop $\leq 1$;

- большой протяженностью атеросклеротического поражения ИЗА;

- многососудистым поражением коронарного русла;

- большим контрольным диаметром ИЗА;

- низкой степенью TIMI и большой тромботической нагрузкой по шкале TIMI thrombus grade до чрескожного коронарного вмешательства (ЧКВ).

В свою очередь, наличие ишемической болезни сердца в семейном анамнезе и артериальная гипертензия снижали шансы развития ФНКК. При этом именно низкая степень TIMI (ОШ=3,83, 95 \% ДИ: $2,77-5,29, \mathrm{p}<0,0001)$ и большая тромботическая нагрузка (ОШ=3,69, 95 \% ДИ: 2,77-5,68, p<0,0001) оказывали наибольшее влияние на вероятность развития ФНКК [22]. Недостатком данной работы является неоднородная подборка статей для метаанализа. Часть исследований выполнены до широкого распространения стентирования и представляют собой анализ пациентов, которым чаще проводилась баллонная ангиопластика, что, по наблюдению некоторых авторов таких работ, является независимым фактором риска развития ФНКК [23]. Помимо этого, в 25 исследованиях ФНКК верифицировался только на основании эпикардиальных 
и субэпикардиальных показателей кровотока после реперфузии. Как было отмечено ранее, такой подход не позволяет диагностировать МВО и, следовательно, не в полной мере соответствует поставленной задаче.

Несомненный интерес представляют исследования, оценивающие морфологию поражения коронарного русла с помощью внутрисосудистого ультразвукового исследования (ВСУЗИ) и оптической когерентной томографии. Эмболы разного размера могут возникать из эпикардиального коронарного тромба и эрозий атеросклеротических бляшек во время первичного ЧКВ, но спонтанная эмболизация возможна и до манипуляции с сосудами [24]. В некоторых случаях ФНКК может быть связан со смещением обструктивного тромба дистальнее пораженного участка. Следует отметить, что это явление не обнаруживается с помощью ангиографии, поэтому требуются другие методы визуализации коронарных артерий, такие как ВСУЗИ и оптическая когерентная томография [25]. Исследование T. Soeda et al. [26], включающее в себя 72 пациента с ИМпST, показало высокую прогностическую ценность липидного индекса при значении >3500 по данным оптической когерентной томографии (площадь под $\mathrm{ROC}$-кривой $=0,77$, $\mathrm{p}=0,002)$ и объем атеросклеротической бляшки по данным внутрисосудистого ультразвукового исследования $>81,5$ \% (площадь под ROC-кривой $=0,70$, $\mathrm{p}=0,002)$. Взаимодействие при многофакторном анализе с остальными данными и отношения шансов для этих показателей авторами продемонстрированы не были. Схожие данные были получены в 2002 г. A. Tanaka et al. [27]. В исследовании, включавшем в себя 100 исследуемых с помощью ВСУЗИ, оценивалась морфология атеросклеротической бляшки. Независимыми предикторами являлись большая площадь поперечного сечения покрышки (ОШ=1,55, 95 \% ДИ: 1,01-2,38) и большое липидное ядро (конгломерат низкоэхогенного материала, покрытый высокоэхогенным слоем) (ОШ=118, 95 \% ДИ: 1,28-11008). Альтернативные результаты на больших выборках получили R. Iijima, M. Endo et al. B paботе R. Ijima et al. [28], основанной на анализе анамнеза, коронароангиографий и данных ВСУЗИ 220 пациентов с ИMпST, независимыми факторами риска развития ФНКК признаны тромбообразование (ОШ=4,53, 95 \% ДИ: 1,03-20,0) и большая протяженность фиброатеромы (ОШ=1,79, 95 \% ДИ: 1,01-3,23). При этом большие липидные ядра намного чаще встречались в группе с ФНКК (65 \% против $37 \%$, p <0,05), однако значимым предиктором не являлись. Согласно результатам исследования M. Endo et al. [29], проанализировавших данные анамнеза, коронароангиографий и ВСУЗИ 170 пациентов с ИMпST, значимыми предикторами ФНКК, по данным многофакторного регрессионного анализа, являются затухание ультразвукового сигнала на длине $\geq 5$ мм (ОШ=20,1, 95 \% ДИ: 5,87-69,0), разрыв бляшки (ОШ $=5,94,95$ \% ДИ: 1,6-21,5) и время боль-баллон (за каждый час) (ОШ=1,26, 95 \% ДИ: $1-1,58)$.

Также ряд авторов подчеркивают важность воспаления, активации тромбоцитов в развитии РСМ в целом и, в частности, ФНКК [30]. Исследование M. Akpek et al. [31] показало, что большой показатель отношения нейтрофилов к лимфоцитам и высокая концентрация C-реактивного белка в плазме крови являются значимыми независимыми предикторами развития no-reflow. При концентрации высокочувствительного С-реактивного белка >5,3 мг/л площадь под ROC-кривой составляла 0,89 (95\% ДИ: 0,86-0,92), а при отношении нейтрофилов к лимфоцитам >3,3-0,80 (95 \% ДИ: 0,75-0,84). Спустя год этой же группой ученых продемонстрирована прогностическая значимость среднего объема тромбоцитов (ОШ=1,075, 95 \% ДИ=1,009-1,543, p=0,38) [32]. Однако недавнее исследование A. Kurtul et al. [33] продемонстрировало значительно большую предсказательную силу отношения среднего объема тромбоцитов к лимфоцитам и отношения тромбоцитов к лимфоцитам в сравнении со средним объемом тромбоцитов. Для прогнозирования ФНКК лучшими показателями для отношения среднего объема тромбоцитов к лимфоцитам $=4,87$ (площадь под ROCкривой $=0,819,95 \%$ ДИ $=0,787-0,850)$, для отношения тромбоцитов к лимфоцитам = 133 (площадь под ROC-кривой $=0,797,95 \%$ ДИ $=0,761-0,833)$, и для среднего объема тромбоцитов $=8,65$ (площадь под $\mathrm{ROC}$-кривой $=0,587,95 \%$ ДИ = 0,544-0,630). Помимо этого, считается, что отношение моноцитов к липопротеинам высокой плотности является маркером воспаления и окислительного стресса при сердечно-сосудистых заболеваниях. S. Balta et al. [34] в исследовании на выборке объемом 600 пациентов c ИMпST установили, что отношение моноцитов к липопротеинам высокой плотности является независимым предиктором феномена no-reflow (при пороге отсечения $=22,5$ площадь под $\mathrm{ROC-кривой}=0,768$, 95 \% ДИ: 0,725-0,812). К сожалению, мультиколлинеарность (корреляция между предикторами, приводящая к неустойчивости их оценок) маркеров воспалительных процессов практически не оценивается авторами данных исследований, и выделить наиболее значимый прогностический показатель, опираясь на представленные данные, затруднительно.

Кроме того, существует гипотеза о значительном влиянии на шансы развития феномена no-reflow постоянного, длительного приема пациентами некоторых групп препаратов до ЧКВ. В ряде исследований подтверждено, что прием В-адреноблокаторов значительно снижал вероятность развития ФНКК. Ретроспективное исследование Wang et al. [35], проведенное на основании анализа 1615 пациентов, показало, что длительный прием В-адреноблокаторов до первичного ЧКВ оказывал значительное протективное воздействие (ОШ=0,594, 95 \% ДИ: 0,394-0,893). Аналогичные результаты были получены в других исследованиях $[31,36]$. Отдельные работы так же сообщают о прогностической ценности длительного приема перед ИМпST ингибиторов ангиотензинпревращающего фермента [37], статинов [38, 39] и блокаторов рецепторов ангиотензина [40]. Однако немало исследований противоречат данным гипотезам, и для их подтверждения требуется большая доказательная база. 


\section{ОБЗОРЫ / REVIEWS}

Большой интерес вызывает построенная J. W. Wang et al. [41] предсказательная шкала развития ФНКК. В результате анализа данных 1776 пациентов с ИMпST авторы выстроили прогностическую модель, основанную на 7 факторах с присвоенными баллами:

1) возраст $\geq 55$ лет $=5$ баллов;

2) время боль-баллон $\geq 4$ ч $=2$ балла;

3) концентрация глюкозы в плазме крови $\geq 12,0$ ммоль/л = 4 балла;

4) количество нейтрофилов в крови $\geq 8,81 \cdot 10^{9}$ ед./л $=8$ баллов;

5) класс острой сердечной недостаточности по Killip 4 = 3 балла;

6) степень TIMI thrombus grade до ЧКВ $\geq 2=5$ баллов;

7) коллатеральный кровоток до ЧКВ по Rent$\mathrm{rop} \leq 1=2$ балла.

При сумме баллов, равной 14, высока вероятность развития ФНКК. Данная предсказательная модель обладает весьма высокой прогностической силой (площадь под ROC кривой $=0,80095$ \% ДИ: 0,772-0,826). В данном исследовании феномен no-reflow верифицировался на основании показателей эпикардиального кровотока (TIMI и MBG), что не отображает состояние микроциркуляторного русла после ЧКВ.

На основании недавнего крупного исследования Y. Li et al. [42], основанного на анализе 1658 пациентов с ИМпSТ, перенесших ЧКВ со стентированием ИЗА, также была построена прогностическая модель развития ФНКК. Данная работа интересна подходом к формированию критериев включения и исключения, нивелирующим вероятность попадания в выборку пациентов, наличие которых заведомо может привести к статистическим ошибкам. Так, критериями включения в исследование были возраст пациентов $\geq 18$ лет; время от начала заболевания $\leq 24$ ч; диагноз «Острый ИМпSТ»; успешная реканализация ИЗА. Критериями исключения из исследования являлись аллергические реакции на антиагреганты, антикоагулянты и йод-содержащие контрастные вещества; противопоказания к антиагрегантной терапии, такие как активное висцеральное кровоизлияние, геморрагический инсульт или ишемический инсульт в течение полугода (включая преходящую ишемическую атаку), расслоение аорты или гематологические заболевания, осложненные нарушениями коагуляции; аортокоронарное шунтирование в анамнезе; патология клапанов сердца и кардиомиопатии; полная блокада левой ножки пучка Гиса, искусственный водитель ритма и другие факторы, приводящие к изменениям сегмента ST на электрокардиограмме; тяжелая печеночная и почечная недостаточность; злокачественные новообразования или аутоиммунные заболевания; тяжелые инфекционные заболевания в недавнем времени. Прогностическая модель, построенная исследователями, включает в себя следующие предикторы:

1) возраст пациента $\geq 65$ лет $=2$ балла;

2) отсутствие приема ингибиторов ангиотензинпревращающего фермента/антагонистов рецепторов ангиотензина II в анамнезе = 1 балл;
3) коллатеральный кровоток до ЧКВ по Rentrop $<2$ степени = 3 балла;

4) степень TIMI thrombus grade до ЧКВ $\geq 4$ степени = 2 балла;

5) диаметр пораженного сосуда $\geq 3,5$ мм = 1 балл;

6) глюкоза плазмы крови $>8$ ммоль/л $=1$ балл.

Однако ROC-анализ построенной математической модели не показал хороших результатов, зона под $\mathrm{ROC}$-кривой $=0,648$ (чувствительность $=42,0 \%$, специфичность $=79,3 \%, \mathrm{p}<0,001)$ в группе разработки модели и 0,637 в группе апробации модели (специфичность $=53,2 \%$, чувствительность $=66,7 \%$, $\mathrm{p}<0,001)$. Порог баллов, при котором был высокий риск развития ФНКК, авторами не указывается [42].

\section{Закцючение}

В заключение следует отметить, что ФНКК является сложным, многофакторным патологическим процессом. Разные подходы к верификации феномена no-reflow, выбору пациентов для включения в исследования, большое число предикторов, часть из которых, вероятно, коррелирует между собой, значительно затрудняют синтез информации по данной проблеме. Однако ряд факторов, такие как степень кровотока в ИЗА, тромботическая нагрузка, активное воспаление, чаще всего встречаются в исследуемых работах и, вероятно, являются ключевыми. Таким образом, несмотря на большое число исследований, посвященных предикторам ФНКК, единого мнения по поводу данной проблемы нет, и она не утрачивает свою важность и актуальность.

\section{Конфликт интересов / Conflict of interest}

Авторы заявили об отсутствии конфликта интересов. / The authors declare no conflict of interest.

\section{Аитература / References}

1. Kloner RA. Does reperfusion injury exist in humans // J. Am. Coll. Cardiol. 1993;(21):537-545. Doi: 10.1016/07351097(93)90700-b.

2. Durante A, Camici PG. Novel insights into an «old» phenomenon: the no reflow // Int. J. Cardiol. 2015;(187):273-280. Doi: 10.1016/j.ijcard.2015.03.359.

3. Schwartz BG, Kloner RA. Coronary no reflow // J. Mol. Cell Cardiol. 2012;52(4):873-882. Doi: 10.1016/j.yjmcc. 2011.06.009

4. Eeckhout E, Kern MJ. The coronary no-reflow phenomenon: a review of mechanisms and therapies // Eur. Heart J. 2001;(22):729-739. Doi: 10.1053/euhj.2000.2172.

5. Синдром реперфузии миокарда: патогенез, клиника, диагностика / Г. Г. Хубулава, А. Н. Шишкевич, С. С. Михайлов, Е. Ю. Бессонов // Вестн. Рос. Военно-мед. акад. 2020. - T. 1, № 69. - C. 196-200. [Khubulava GG, Shishkevich AN, Mihajlov SS, Bessonov EJu. Sindrom reperfuzii miokarda: patogenez, klinika, diagnostika // Vestnik Rossijskoj Voennomedicinskoj akademii. 2020;1(69):196-200. (In Russ.)].

6. Фролов А. А., Починка И. Г., Шахов Б. Е. и др. Феномен коронарной микрососудистой обструкции (no-reflow) при проведении чрескожных коронарных вмешательств у пациентов с инфарктом миокарда // Патология кровообращения и кардиохирургия. - 2020. T. 24, № 1. - С. 18-27. [Frolov AA, Pochinka IG, Shahov BE. et al. Fenomen koronarnoj mikrososudistoj obstrukcii (no-reflow) pri provedenii chreskozhnyh koronarnyh vmeshatel'stv u pacientov 
s infarktom miokarda // Patologija krovoobrashhenija $i$ kardiohirurgija. 2020;24(1):18-27. (In Russ.)].

7. Niccoli G, Cosentino N, Spaziani C. et al. No-reflow: incidence and detection in the cath-lab // Curr. Pharm. Des. 2013;(19):4564-4575. Doi: 10.2174/1381612811319250005.

8. Niccoli G, Scalone G, Lerman A. et al. Coronary microvascular obstruction in acute myocardial infarction // European Heart J. 2016;37(13):1024-1033. Doi: 10.1093/ eurheartj/ehv484.

9. Morishima I, Sone T, Okumura K. et al. Angiographic no-reflow phenomenon as a predictor of adverse long-term outcome in patients treated with percutaneous transluminal coronary angioplasty for first acute myocardial infarction // J. Am. Coll. Cadiol. 2000;36(4):1202-1209. Doi: 10.1016/ s0735-1097(00)00865-2.

10. Dong-bao L, Qi H, Zhi L. et al. Predictors and longterm prognosis of angiographic slow/no-reflow phenomenon during emergency percutaneous coronary intervention for ST-elevated acute myocardial infarction // Clin. Cardiol. 2010;33(12):7-12. Doi: 10.1002/clc.20634.

11. Mehta RH, Harjai KJ, Boura J. et al. Prognostic significance of transient no-reflow during primary percutaneous coronary intervention for ST-elevation acute myocardial infarction // Am. J. Cardiol. 2003;92(12):1445-1447. Doi: 10.1016/j.amjcard.2003.08.056.

12. de Waha $S$, Patel MR, Granger CB. et al. Relationship between microvascular obstruction and adverse events following primary percutaneous coronary intervention for STsegment elevation myocardial infarction: an individual patient data pooled analysis from seven randomized trials // Eur. Heart J. 2017;38(47):3502-3510. Doi: 10.1093/eurheartj/ehx414.

13. Al Azzoni A, Lamelos P, Marsden T. Incidence and predictors of no reflow phenomenon: insights from the total trial // Journal of the American College of Cardiology. 2017;69(11):1179. Doi: 10.1016/s0735-1097(17)34568-0.

14. Cenko E, Ricci B, Kedev S. et al. The no-reflow phenomenon in the young and in the elderly//Int. J. of Cardiology. 2016;(222):1122-1128. Doi: 10.1016/j.ijcard.2016.07.209.

15. Lerman A, Holmes DR, Herrman J. et al. Microcirculatory dysfunction in ST-elevation myocardial infarction: cause, consequence, or both // Eur. Heart J. 2007;28(7):788-797. Doi: 10.1093/eurheartj/ehl501.

16. Britten MB, Zeiher AM, Schächinger V. Microvascular dysfunction in angiographically normal or mildly diseased coronary arteries predicts adverse cardiovascular long-term outcome. Coron // Artery Dis. 2004;15(5):259-264. Doi: 10.1097/01.mca.0000134590.99841.81.

17. Nijveldt R, van der Vleuten PA, Hirsch A. et al. Early Electrocardiographic Findings and MR Imaging-Verified Microvascular Injury and Myocardial Infarct Size // JACC: Cardiovascular Imaging. 2009:2(10):1187-1194. Doi: 10.1016/j. jcmg.2009.06.008.

18. Niccoli G, Burzotta F, Galiuto L. et al. Myocardial noreflow in humans // J. Am. Coll. Cardiol. 2009;54(4):281-292. Doi: 10.1016/j.jacc.2009.03.054.

19. Sorajja P, Gersh BJ, Costantini C. et. al. Combined prognostic utility of ST-segment recovery and myocardial blush after primary percutaneous coronary intervention in acute myocardial infarction // Eur. Heart J. 2005;26(7):667-674. Doi: 10.1093/eurheartj/ehil67.

20. Bolognese L, Carrabba N, Parodi G. et al. Impact of microvascular dysfunction on left ventricular remodeling and longterm clinical outcome after primary coronary angioplasty for acute myocardial infarction // Circulation. 2004;109(9):11211126. Doi: 10.1161/01.cir.0000118496.44135.a7.

21. Wu KC, Zerhouni EA, Judd RM. et al. Prognostic significance of microvascular obstruction by magnetic resonance imaging in patients with acute myocardial infarction // Circulation. 1998;97(8):765-772. Doi: 10.1161/01.cir.97.8.765.

22. Fajar JK, Herianshyah T, Rohman MS. The predictors of no reflow phenomenon after percutaneous coronary intervention in patients with ST elevation myocardial infarction: A meta-analysis // Indian Heart Journal. 2018;70(3):406-418. Doi: 10.1016/j.ihj.2018.01.032.

23. Kirma C, Izgi A, Dundar C. et al. Clinical and Procedural Predictors of No-Reflow Phenomenon After Primary Percutaneous Coronary Interventions // Circulation Journal. 2008;72(5):716-721. Doi: 10.1253/circj.72.716.

24. Sakai S, Mizuno K, Tomimura M. et al. Visualized plaque debris as a cause of distal embolization after percutaneous coronary intervention in patients with unstable angina // Catheter Cardiovasc. Interv. 2002;(55):113-117. Doi: 10. $1002 /$ ccd.10070.

25. den Heijer P, Foley DP, Escaned J. et al. Angioscopic versus angiographic detection of intimal dissection and intracoronary thrombus // J. Am. Coll. Cardiol. 1994;24(3):649654. Doi: 10.1016/0735-1097(94)90010-8.

26. Soeda T, Higuma T, Abe N. et al. Morphological predictors for no reflow phenomenon after primary percutaneous coronary intervention in patients with STsegment elevation myocardial infarction caused by plaque rupture // Eur. Heart J. Cardiovasc. Imaging. 2017;18(1):103-110. Doi: 10.1093/ ehjci/jev341.

27. Tanaka A, Kawarabayashi T, Nishibori Y. et al. No-Reflow Phenomenon and Lesion Morphology in Patients With Acute Myocardial Infarction // Circulation. 2002;105(18):21482152. Doi: 10.1161/01.cir.0000015697.59592.07.

28. Iijima R, Shinji H, Ikeda N. et al. Comparison of coronary crterial finding by intravascular ultrasound in patients with «transient no-reflow» versus «reflow» during percutaneous coronary intervention in acute coronary syndrome // Am. J. Cardiol. 2006;97(1):29-33. Doi: 10.1016/j. amjcard.2005.07.104.

29. Endo M, Hibi K, Shimizu T. et al. Impact of ultrasound attenuation and plaque rupture as detected by intravascular ultrasound on the incidence of no-reflow phenomenon after percutaneous coronary intervention in st-segment elevation myocardial infarction // JACC Cardiovasc. Interv. 2010;3(5):540-549. Doi: 10.1016/j.jcin.2010.01.015.

30. Francischetti I, Moreno JB, Scholz M. et al. Leukocytes and the inflammatory response in ischemia-reperfusion injury //Rev. Bras. Cir. Cardiovasc. 2010;25(4):575-584. Doi: 10. 1590/s0102-76382010000400023.

31. Akpek M, Kaya MG, Lam YY. et al. Relation of neutrophil/ lymphocyte ratio to coronary flow to in-hospital major adverse cardiac events in patients with st-elevated myocardial infarction undergoing primary coronary intervention // Am. J. Cardiol. 2012;110(5):621-627. Doi: 10.1016/j.amjcard. 2012.04.041.

32. Celik T, Kaya MG, Akpek M. et al. Predictive value of admission platelet volume indices for in-hospital major adverse cardiovascular events in acute st-segment elevation myocardial infarction // Angiology. 2013;66(2):155-162. Doi: 10.1177/0003319713513493.

33. Kurtul A, Acikgoz SK. Usefulness of mean platelet volume-to-lymphocyte ratio for predicting angiographic noreflow and short-term prognosis after primary percutaneous coronary intervention in patients with st-segment elevation myocardial infarction // Am. J. Cardiol. 2017;120(4)534-541. Doi: 10.1016/j.amjcard.2017.05.020.

34. Balta $S$, Celik T, Ozturk C. et al. The relation between monocyte to HDL ratio and no-reflow phenomenon in the patients with acute ST-segment elevation myocardial infarction // The American Journal of Emergency Medicine. 2016; 34(8):1542-1547. Doi: 10.1016/j.ajem.2016.05.031. 


\section{ОБЗОРЫ / REVIEWS}

35. Wang J, Chen Y, Wang C. et al. The impact of preprimary percutaneous coronary intervention $\beta$ blocker use on the no-reflow phenomenon in patients with acute myocardial infarction // Chin. J. Cardiol. 2014;42(10):822-826.

36. Celik T, Kaya MG, Akpek M. Does serum bilirubin level on admission predict timi flow grade and in-hospital MACE in patients with STEMI undergoing primary PCI// Angiology. 2014;65(3):198-204. Doi: 10.1177/0003319712474948.

37. Zhao J, Yang Y, Zhang Y. et al. Chronic pretreatment of ACEI reduces no-reflow in patients with acute myocardial infarction treated with primary angioplasty // Clin. Cardiol. 2007;30(3):130-134. Doi: 10.1002/clc.20060.

38. Iwakura K, Ito H, Kawano S. et al. Chronic pre-treatment of statins is associated with the reduction of the no-reflow phenomenon in the patients with reperfused acute myocardial infarction // Eur Heart J. 2006;27(5):534-539. Doi: 10.1093/ eurheartj/ehi715.

39. Kim JS, Kim J, Choi D. et al. Efficacy of high-dose atorvastatin loading before primary percutaneous coronary intervention in ST-segment elevation myocardial infarction: the STATIN STEMI trial // JACC Cardiovasc Interv. 2010; 3(3):332-339. Doi: 10.1016/j.jcin.2009.11.021.

40. Hu T, Wang HC, Wang RT. et al. Effect of chronic pretreatment of angiotensin-converting receptor blocker on no-reflow phenomenon in patients with acute myocardial infarction undergoing percutaneous coronary intervention // Cardiovascular Therapeutics. 2013;31(3):7-11. Doi: 10.1111/j.17555922.2012.00314.x.

41. Wang JW, Zhou ZQ, Chen YD. et al. A risk score for no reflow in patients with ST-segment elevation myocardial infarction after primary percutaneous coronary intervention // Clinical Cardiology. 2015;38(4):208-215. Doi:10.1002/ clc. 22376.

42. LiY, Hongliang C, Yali L. et al. Prediction of no-reflow phenomenon in patients treated with primary percutaneous coronary intervention for ST-segment elevation myocardial infarction. Medicine (Baltimore). 2020;99(26):E20152. Doi: 10.1097/MD.0000000000020152.

\section{Информация об авторах}

Хубулава Геннахий Григорьевич - $\Delta-p$ мед. наук, профессор, академик РАН, зав. кафедрой и клиникой хирургии (усовершенствования врачей) № 1, Военно-медицинская академия им. С. М. Кирова, Санкт-Петербург, Россия, e-mail: as015@rambler.ru.

Козмов Киримл Аенарович - $\Delta-p$ мед. наук, профрессор, профрессор кафредры хирургии (усовершенствования врачей) № 1, Военно-медицинская академия им. С. М. Кирова, СанктПетербург, Россия, e-mail: kozlov_kl@mail.ru.

Шишкевич Андрей Николаевич - $\Delta$-р мед. наук, начальник рентгенохирургического отАеления клиники хирургии (усовершенствования врачей) № 1, Военно-медицинская ака- демия им. С. М. Кирова, Санкт-Петербург, Россия, e-mail: shishkevich50@mail.ru.

Михайлов Сергей Сергеевич - канА. меА. наук, старший ординатор рентгенохирургического отделения клиники хирургии (усовершенствования врачей) №1, Военно-медицинская акацемия им. С. М. Кирова, Санкт-Петербург, Россия, e-mail: mikhailov.vma@gmail.com.

Бессонов Евгений Юрьевич - ординатор рентгенохирургического отАеления клиники хирургии (усовершенствования врачей) № 1, Военно-медицинская академия им. С. М. Кирова, Санкт-Петербург, Россия, e-mail: ewgenijbessonov@ yandex.ru.

Токарев Павел Александрович - врач-PXM М клиники хирургии (усовершенствования врачей) № 1, Военно-медицинская акацемия им. С. М. Кирова, Санкт-Петербург, Россия.

Пачков Амитрий Алексеевич - врач - анестезиолог-реаниматолог клиники хирургии (усовершенствования врачей) № 1, Военно-медицинская академия им. С. М. Кирова, СанктПетербург, Россия, e-mail: pachkov.dmitriy@gmail.ru.

Бобровская Екатерина Евгеньевна - кан $\Delta$. ме $\Delta$. наук, преподаватель кафедры хирургии (усовершенствования врачей) № 1, Военно-медицинская акацемия им. С. М. Кирова, СанктПетербург, Россия.

\section{Authors information}

Khubulava Gennady G. - MD, Professor, Academician the Russian Academy of Sciences, Head of the Departmentand Clinic of Surgery (Improvements of Doctors) № 1, Military Medical Academy, Saint Petersburg, Russia, e-mail: as015@rambler.ru.

Kozlov Kirill L. - MD, professor, professor of the Departmentand Clinic of Surgery (Improvements of Doctors) № 1, Military Medical Academy, Saint Petersburg, Russia, e-mail: kozlov_kl@mail.ru.

Shishkevich Andrey N. - MD, head of the X-ray surgical department of the Surgery Clinic (Improvements of Doctors) № 1 Military Medical Academy, Saint Petersburg, Russia, e-mail: shishkevich50@mail.ru.

Mikhaylov Sergey S. - Ph. D., senior resident of the X-ray surgery department of Surgery Clinic (Improvements of Doctors) № 1, Military Medical Academy, Saint Petersburg, Russia, e-mail: mikhailov.vma@gmail.com.

Bessonov Evgeny Yu. - resident of the X-ray surgical department of the Surgery Clinic (Improvements of Doctors) № 1 , Military Medical Academy, Saint Petersburg, Russia, e-mail: ewgenijbessonov@yandex.ru.

Tokarev Pavel A. - X-ray surgeon of the Surgery Clinic (Improvements of Doctors) № 1 of the Military Medical Academy, Saint Petersburg, Russia.

Pachkov Dmitry A. - anesthesiologist-resuscitator of the Surgery Clinic (Improvements of Doctors) № 1, Military Medical Academy, Saint Petersburg, Russia, e-mail: pachkov.dmitriy@ gmail.ru.

Bobrovskaya Ekaterina E. - Ph. D., lecturer of the department of the Surgery Clinic (Improvements of Doctors) № 1 of the Military Medical Academy, Saint Petersburg, Russia. 